\title{
Modeling Interactions within and between Peptide Amphiphile Supramolecular Filaments
}

Ivan R. Sasselli, ${ }^{1,2}$ Zois Syrgiannis, ${ }^{1,2}$ Nicholas A. Sather, ${ }^{1,3}$ Liam C. Palmer, ${ }^{1,2}$ Samuel I. Stupp. ${ }^{1,2,3,4,5,{ }^{*}}$

${ }^{1}$ Simpson Querrey Institute for BioNanotechnology, Northwestern University, 303 East Superior Street, $11^{\text {th }}$ floor, Chicago, IL 60611, USA

${ }^{2}$ Department of Chemistry, Northwestern University, 2145 Sheridan Road, Evanston, IL 60208, USA.

${ }^{3}$ Department of Materials Science and Engineering, Northwestern University, 2220 Campus Drive, Evanston, IL 60208, USA.

${ }^{4}$ Department of Medicine, Northwestern University, 676 N St. Clair, Chicago, IL 60611, USA

${ }^{5}$ Department of Biomedical Engineering, Northwestern University, 2145 Sheridan Road, Evanston, IL 60208, USA.

supramolecular polymer, self-assembly, peptide amphiphile, superstructure, nanofibers, molecular dynamics, coarse-grained simulations, supramolecular filaments, supramolecular biomaterials

* s-stupp@,northwestern.edu 


\section{Contents}

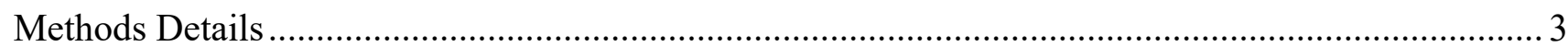

Coarse Grained Molecular Dynamics Simulations.............................................................. 3

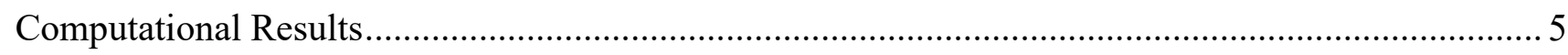

Simulations Starting from Randomly Disposed Molecules ...................................................... 5

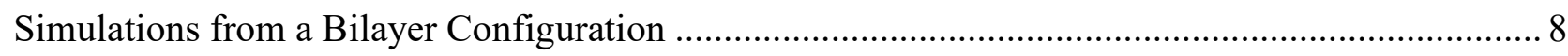

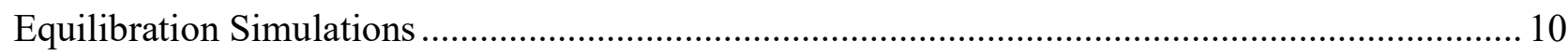

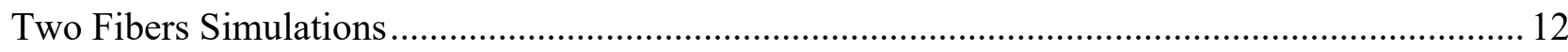

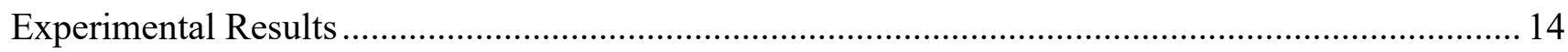

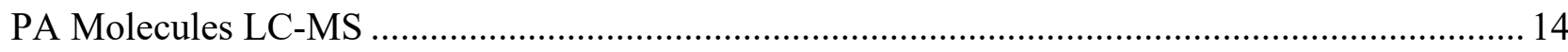

AFM

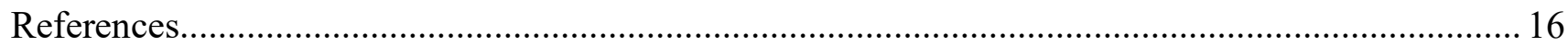




\section{Methods Details}

\section{Coarse Grained Molecular Dynamics Simulations}

The atomistic PA structures were built in Avogadro ${ }^{1}$ and then mapped to MARTINI $2.2^{2-4}$ force field using a modified version of the martinize code to add the aliphatic tail. ${ }^{4-5}$ Coiled coil secondary structure was used in all cases. The systems were investigated with $0,1,1.5,2$ and 3 charges/PA as way of investigating potential changes with $\mathrm{pH}$. Charges were always placed the furthest from the aliphatic tail and the 1.5 was achieved with 50:50 populations of 1 and 2. Self-assembly systems where built using insert-molecules of GROMACS 5.0.4..$^{6-7}$ to insert 300 or 600 molecules into a cubic box of $17.1 \mathrm{~nm}$ per side. This gives concentrations of $100 \mathrm{mM}$ and $200 \mathrm{mM}$, respectively, which is consistent with a tenfold increase in concentration systems that is frequently used to favor self-assembly over practical simulation timescales. ${ }^{8-11}$ For the fiber equilibrations an already assembled fiber is used and placed on the center of a box of the same dimensions. The two fibers systems are built with GROMACS genconf to situate the surfaces of the two fibers within $1 \mathrm{~nm}$. The box size is $y=z=$ fiber length and $x=27 \mathrm{~nm}$. Both, the fiber equilibrations and two fibers systems have the fibers situated along the $z$-axis. The bilayer configurations are generated using the insane code. ${ }^{12}$ All the systems were solvated, and enough ions were added $\left(\mathrm{Na}^{+}\right)$ to ensure the neutrality of the system. All visualizations were rendered using VMD. ${ }^{13}$

All CG-MD simulations and analysis were carried out in GROMACS 5.0.4. using the MARTINI 2.2 force field. Non-bonded interactions were modelled with $1.1 \mathrm{~nm}$ cut-off using potential shift for Lennard-Jones and reaction field with a relative dielectric constant of 15 for electrostatics. ${ }^{14}$ All systems were minimized for 5000 steps or until forces converged bellow $2000 \mathrm{pN}$. MD simulations were run in NPT ensemble with a 25 fs times step. Simulations were run for $5 \cdot 10^{7}$ steps which corresponds to $5 \mu \mathrm{s},{ }^{2}$ except those with two fibers that were twice as long $(10 \mu \mathrm{s})$. Berendsen algorithm was used to keep the pressure constant $\left(1 \mathrm{bar}, \tau_{\mathrm{P}}=3 \mathrm{ps}\right)^{15}$ and $\mathrm{V}$-rescale for the temperature $\left(303 \mathrm{~K}, \tau_{\mathrm{T}}=1 \mathrm{ps}\right)^{16}$. Isotropic conditions were used for all but the fiber equilibration and simulations using a bilayer configuration, which were carried out in semiisotropic conditions (decoupling $\mathrm{z}$ from $\mathrm{x}$ and $\mathrm{y}$ ). The bilayer simulations included a preequilibration $(5 \mu \mathrm{s})$ in isotropic conditions. 
a)
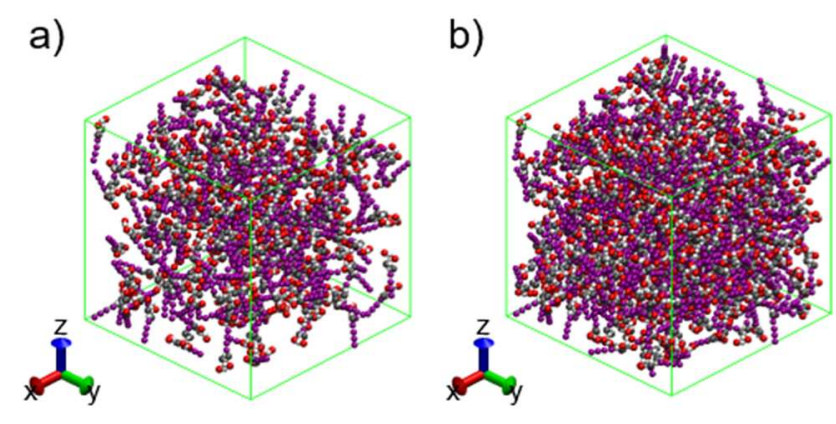

e)

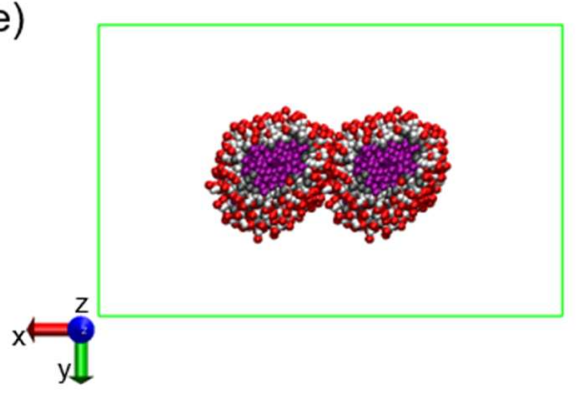

c)

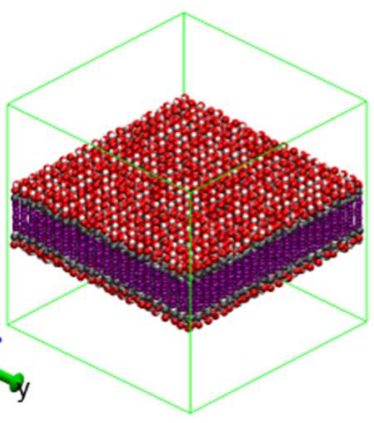

d)

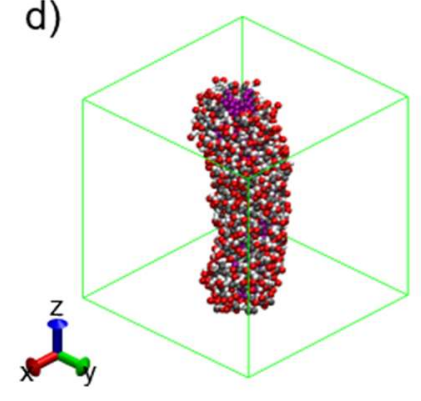

f)

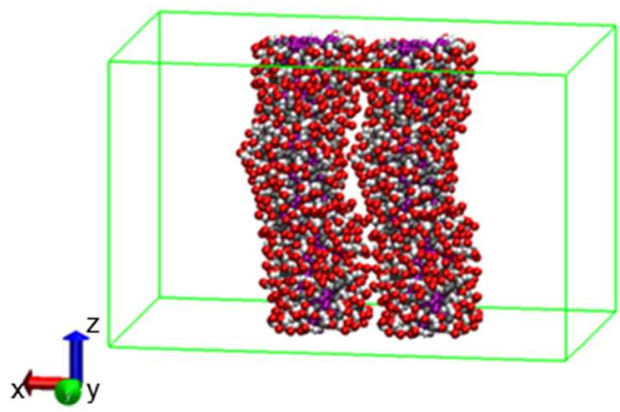

Figure S1. Initial structures of the different simulations: (a) 300 and (b) 600 randomly disposed molecules, (c) bilayer and (d) fiber configurations, and (e) top and (f) front view of the two fibers. Structures are shown with the $\mathrm{C}_{16}$ in purple, peptide backbone in white, hydrophobic side chains in grey, and hydrophilic side chains in red. Simulation box is shown in green. Water and ions are omitted for clarity. 
Simulations Starting from Randomly Disposed Molecules

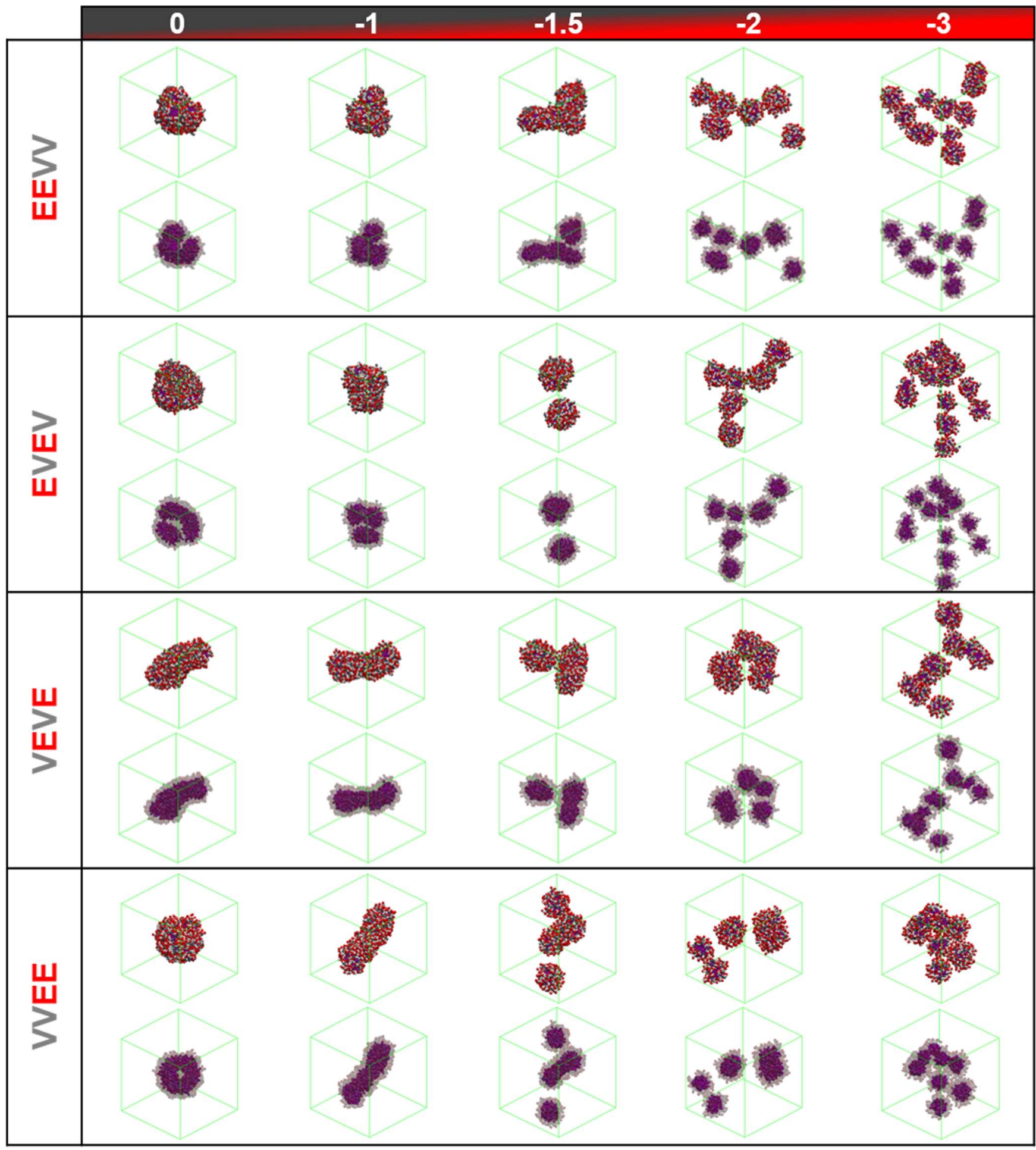

Figure S2. Final structures of the simulations starting from 300 randomly disposed PA molecules for the four isomers EEVV, EVEV, VEVE and VVEE, at different charge/PA. Structures are shown with the aliphatic tail in purple, peptide backbone in white, hydrophobic side chains in grey, and hydrophilic side chains in red. Simulation 
box is shown in green. Water and ions are omitted for clarity. Bottom row for each PA shows peptide semitransparent.

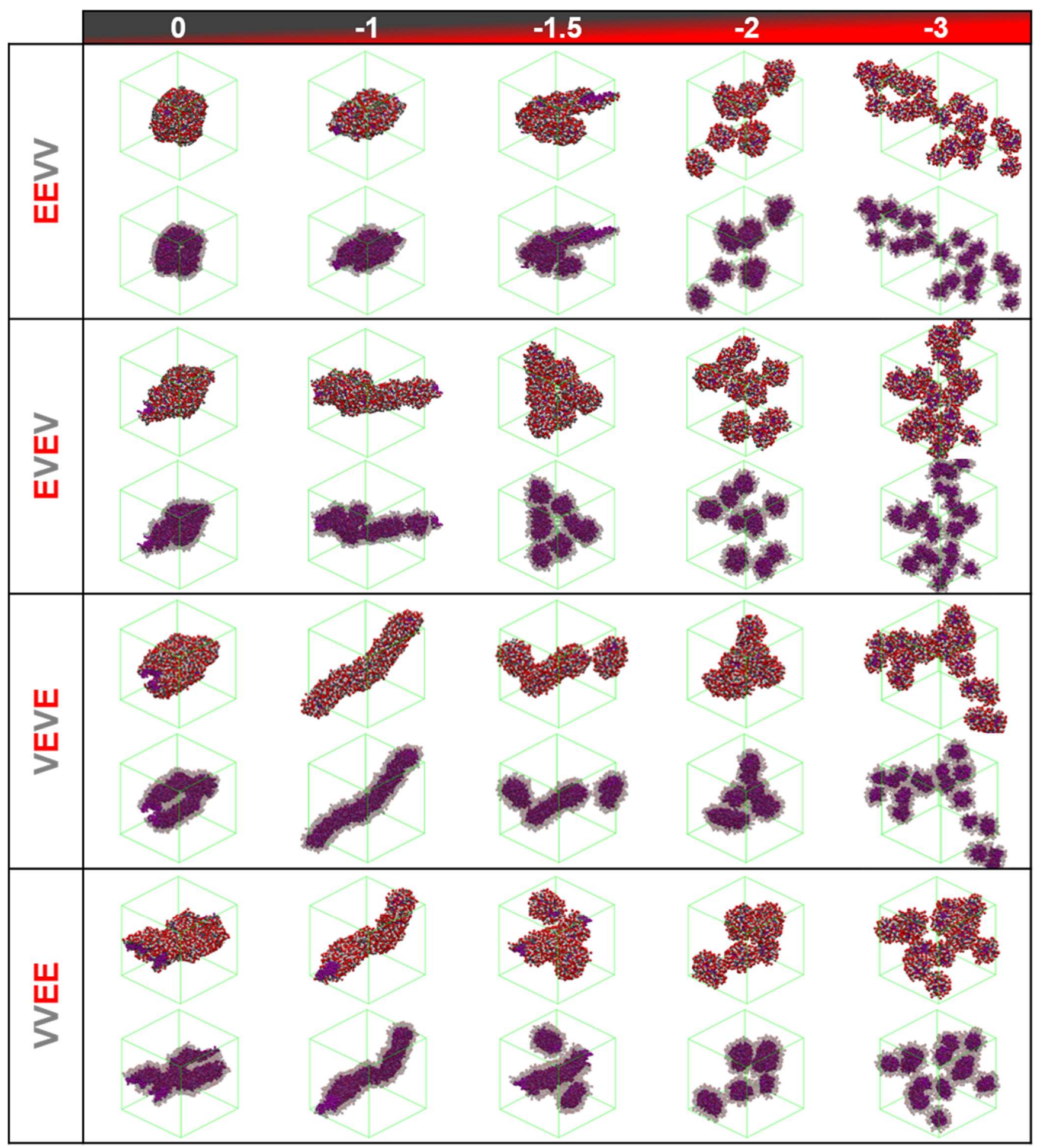

Figure S3. Final structures of the simulations starting from 600 randomly disposed molecules for the four isomers EEVV, EVEV, VEVE and VVEE at different charge/PA. Structures are shown with the aliphatic tail in purple, peptide backbone in white, hydrophobic side chains in grey, and hydrophilic side chains in red. Simulation box is shown in green. Water and ions are omitted for clarity. Bottom row for each PA shows peptide semi-transparent. 
a)

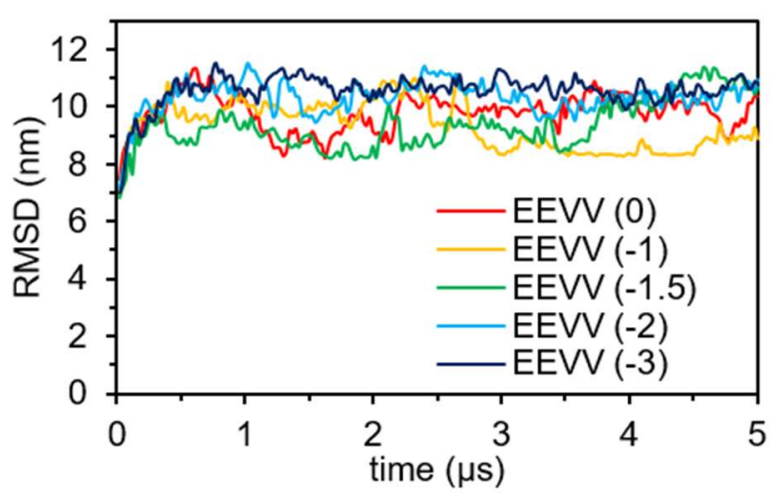

c)

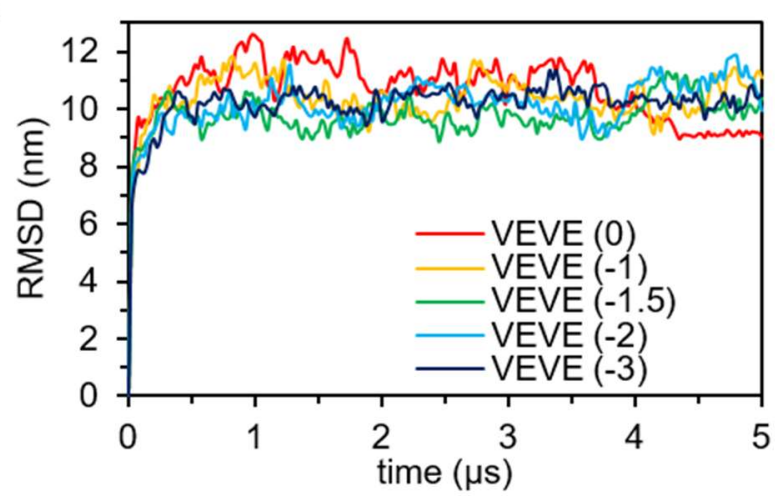

b)

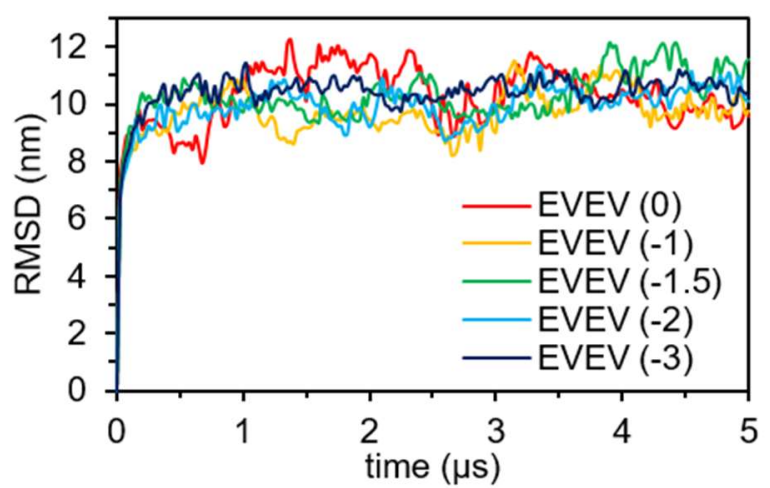

d)

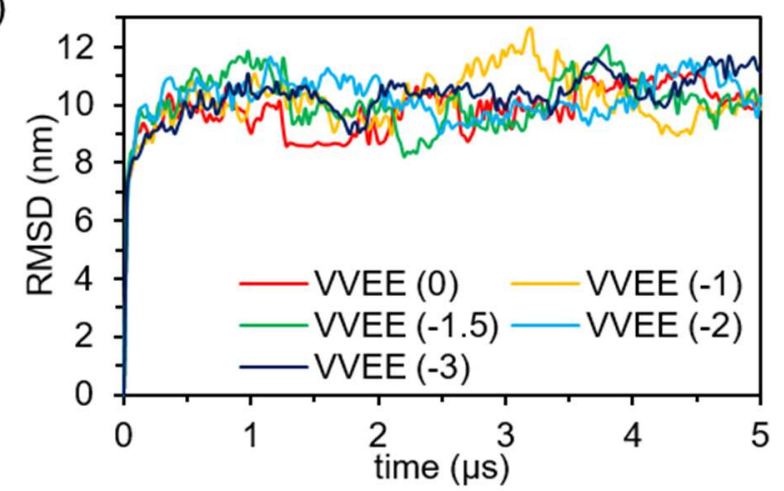

Figure S4. RMSD analysis of the simulations from 300 randomly disposed molecules for the tetrapeptide isomers (a) EEVV, (b) EVEV, (c) VEVE and (d) VVEE, at different charge/PA.

a)

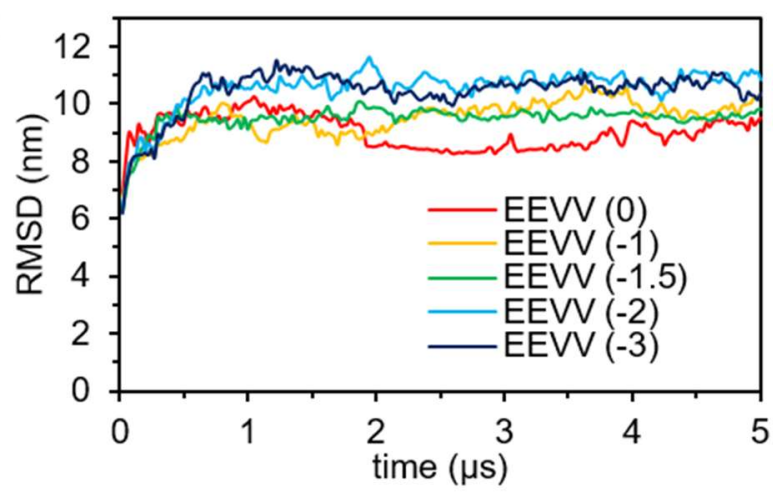

c)

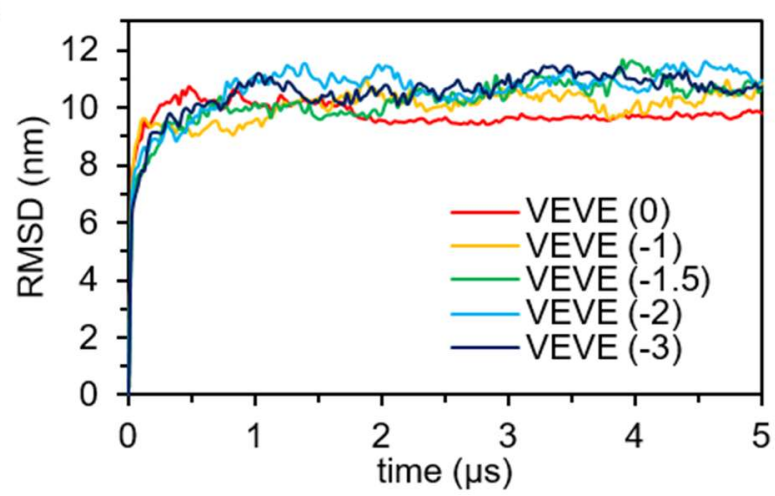

b)

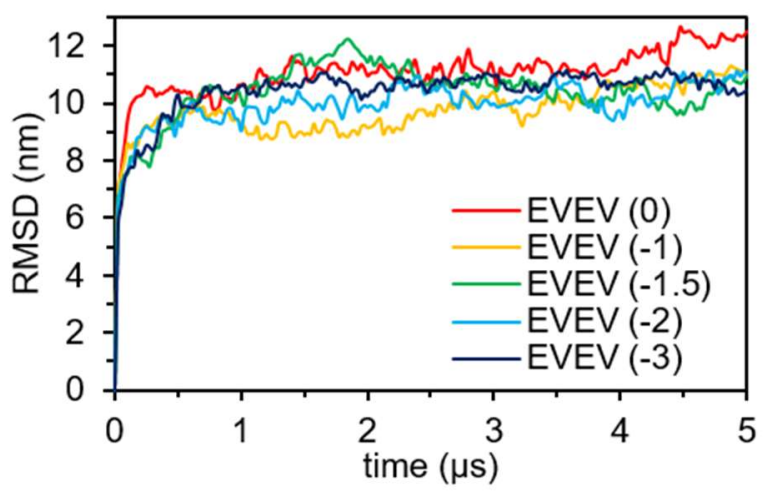

d)

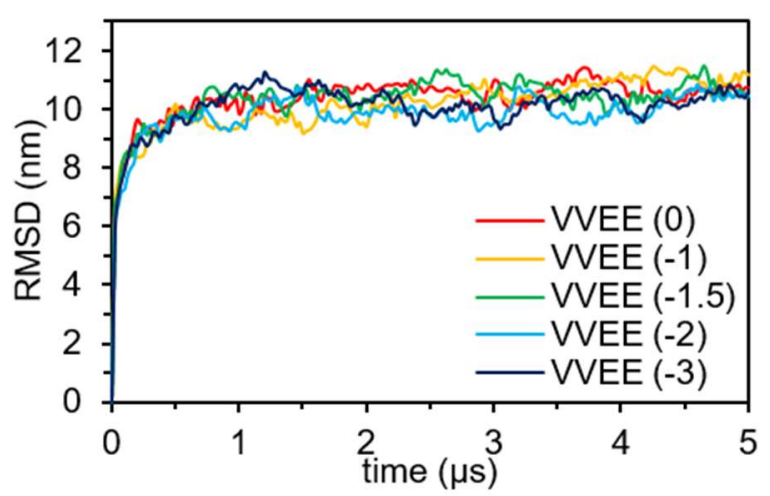

Figure S5. RMSD analysis of the simulations starting from 600 randomly disposed molecules for the tetrapeptide isomers (a) EEVV, (b) EVEV, (c) VEVE and (d) VVEE, at different charge/PA. 


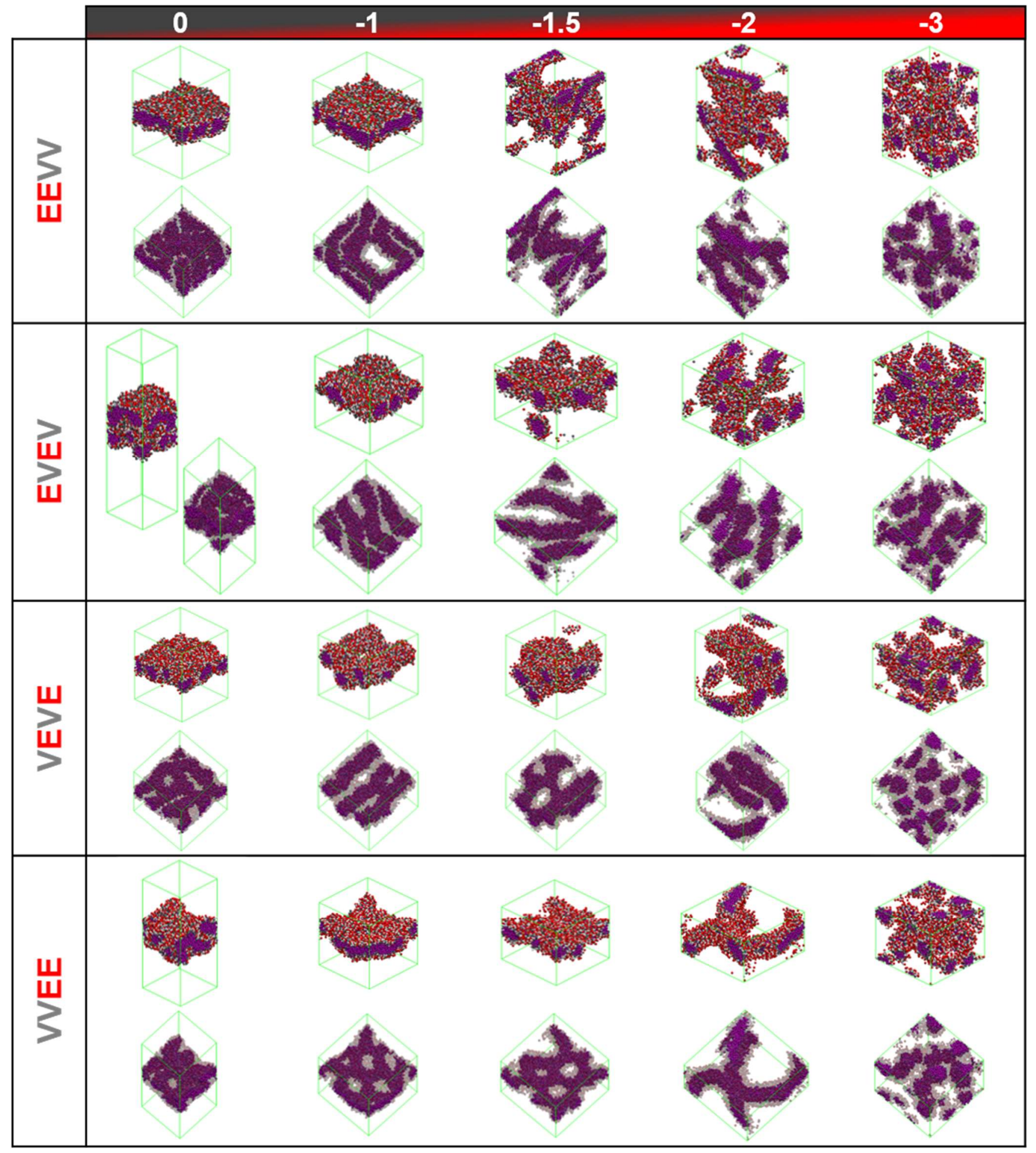

Figure S6. Final structures of the simulations starting from a bilayer configuration for the four isomers EEVV, EVEV, VEVE and VVEE at different charge/PA. Structures are shown with the aliphatic tail in purple, peptide backbone in white, hydrophobic side chains in grey, and hydrophilic side chains in red. Simulation box is shown in green. Water and ions are omitted for clarity. Bottom row for each PA shows peptide semi-transparent. 
a)

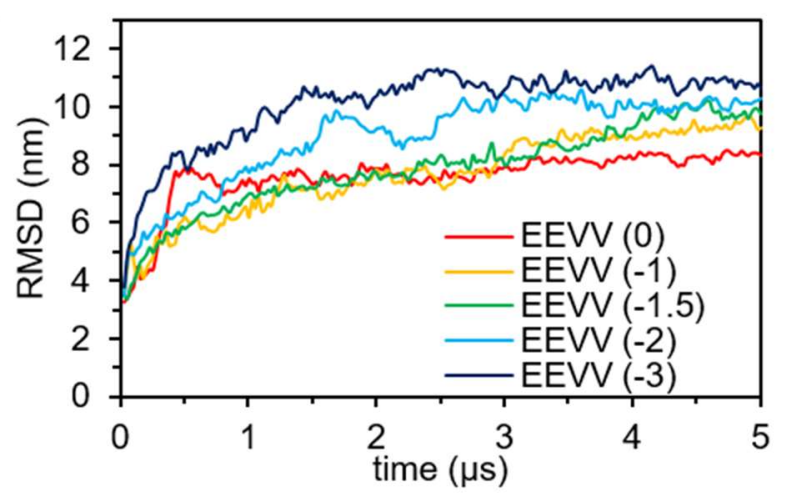

c)

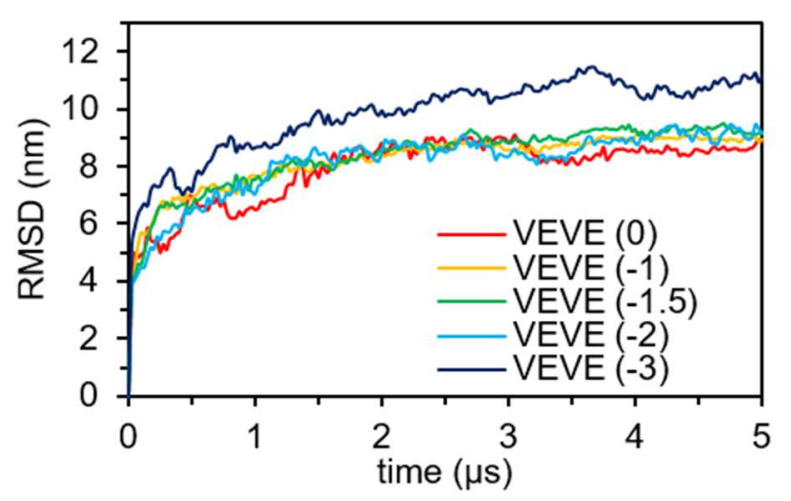

b)

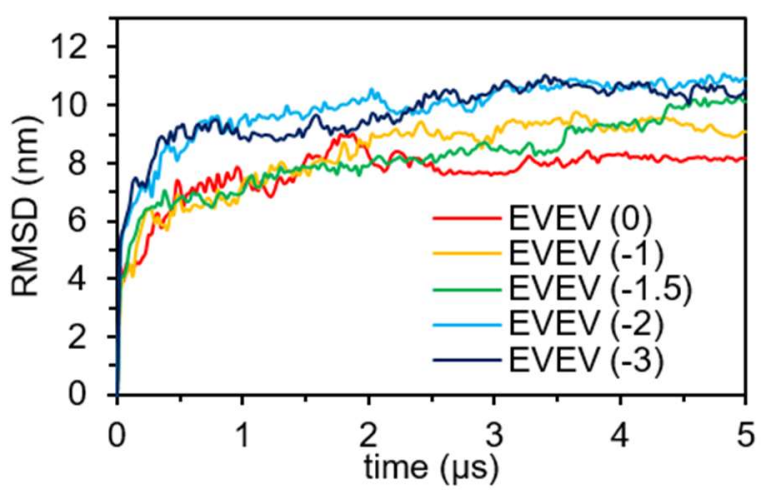

d)

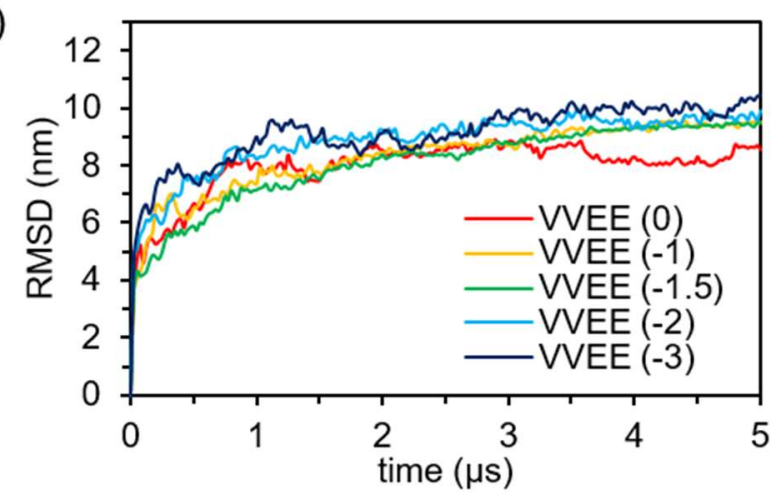

Figure S7. RMSD analysis of the simulations starting from a bilayer configuration for the four tetrapeptide isomers (a) EEVV, (b) EVEV, (c) VEVE and (d) VVEE, at different charge/PA. 


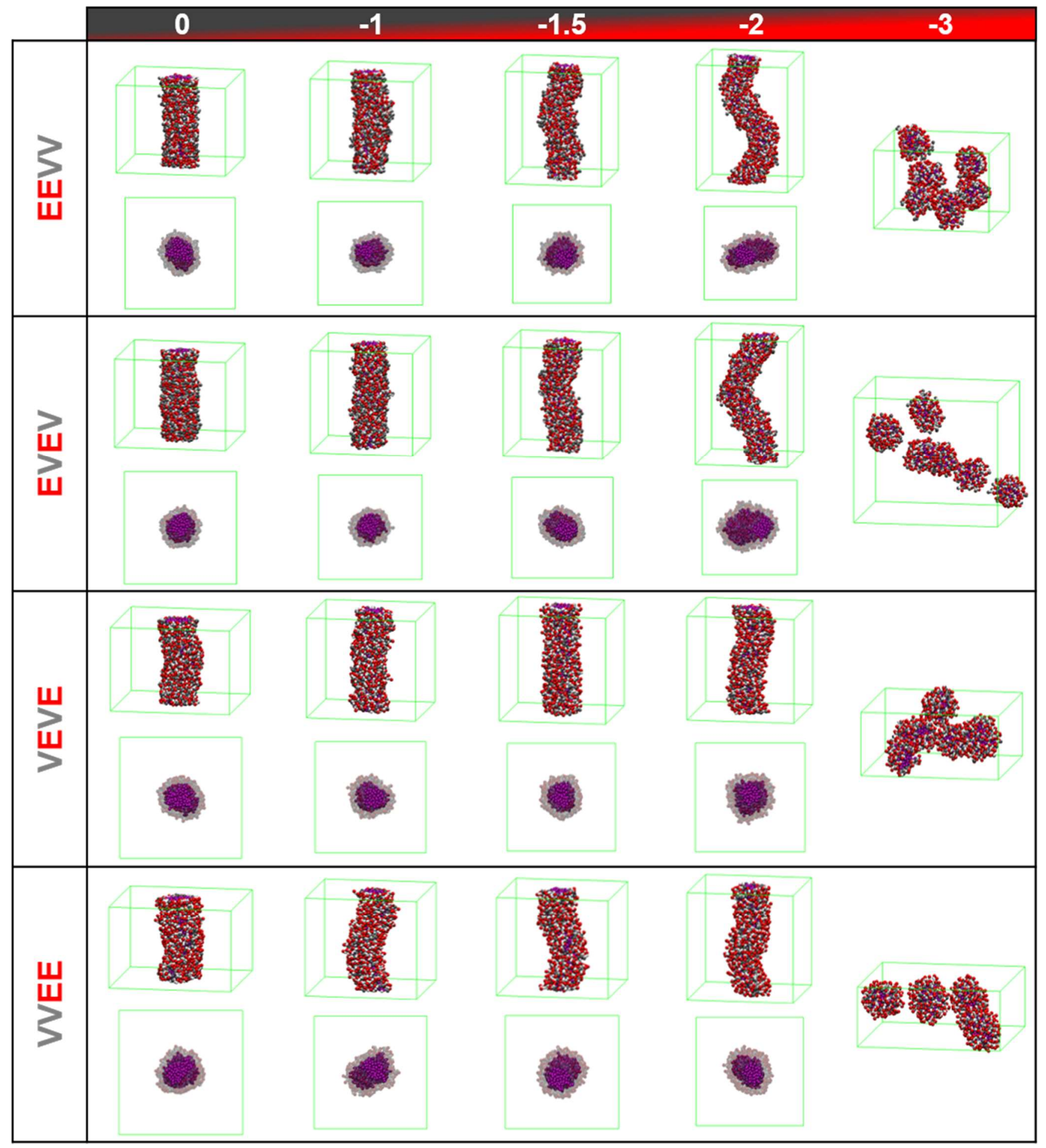

Figure S8. Final structures of the fiber equilibration simulations for the four isomers EEVV, EVEV, VEVE and VVEE at different charge/PA. Structures are shown with the aliphatic tail in purple, peptide backbone in white, hydrophobic side chains in grey, and hydrophilic side chains in red. Simulation box is shown in green. Water and ions are omitted for clarity. Bottom row for each PA shows top view with peptide semi-transparent. 
a)

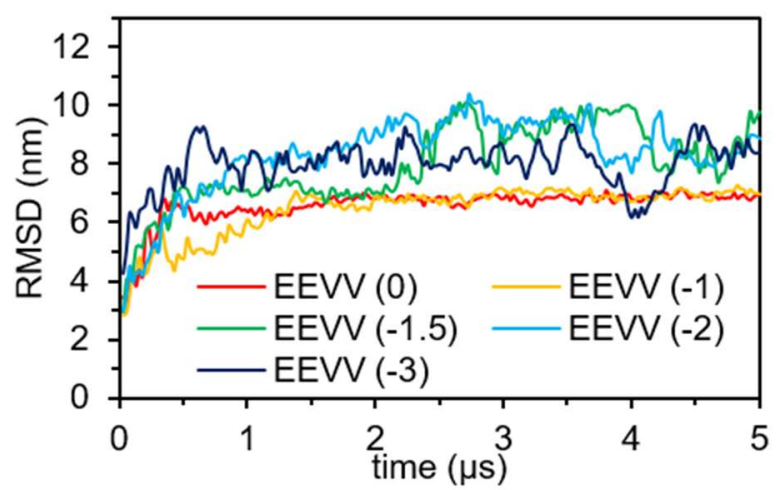

c)

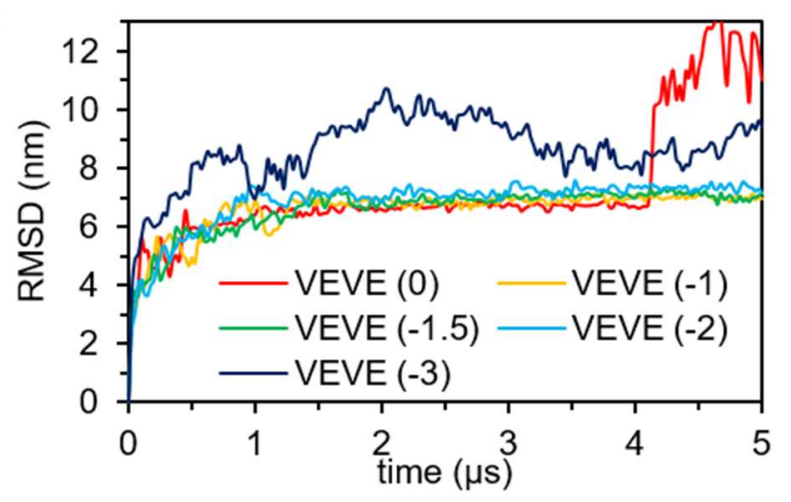

b)

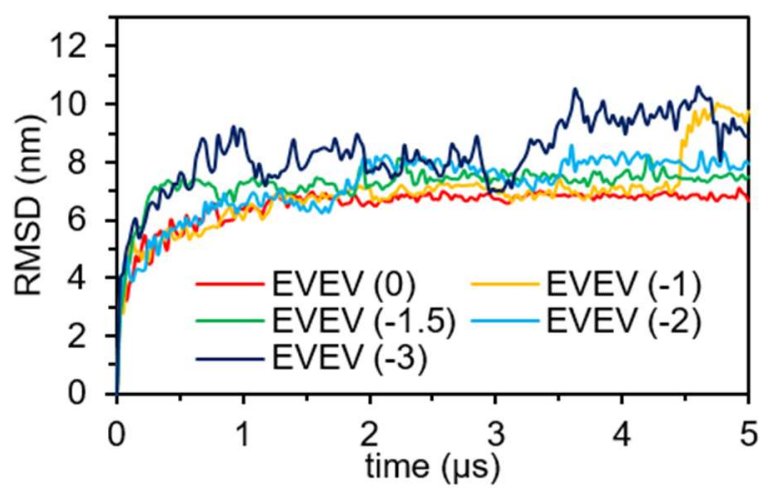

d)

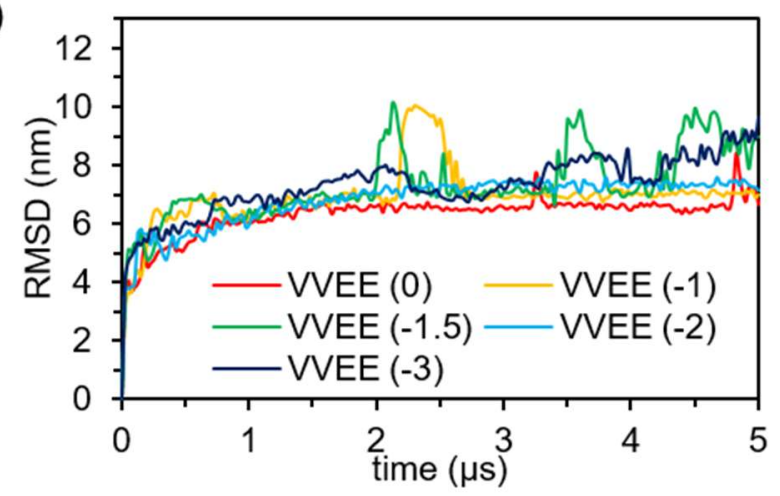

Figure S9. RMSD analysis of the fiber equilibration simulations for the four tetrapeptide isomers (a) EEVV, (b) EVEV, (c) VEVE and (d) VVEE, at different charge/PA.

a)

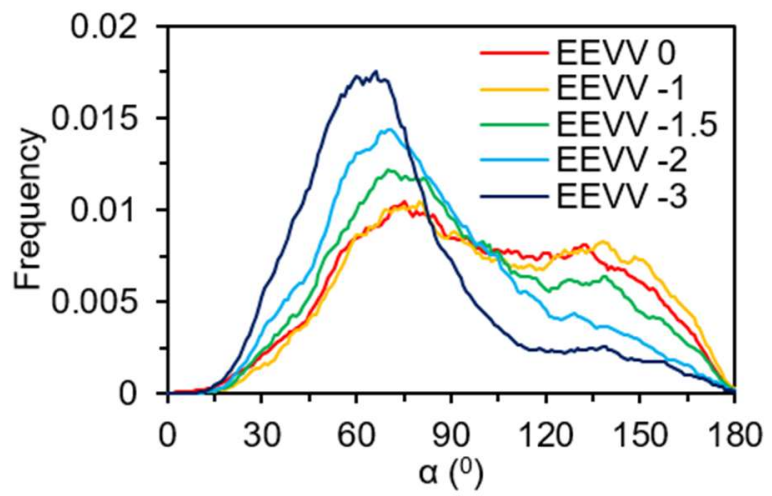

C)

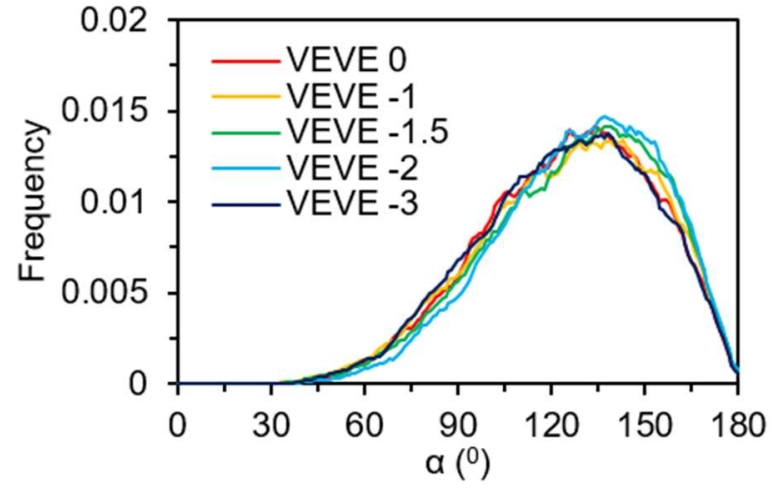

b)

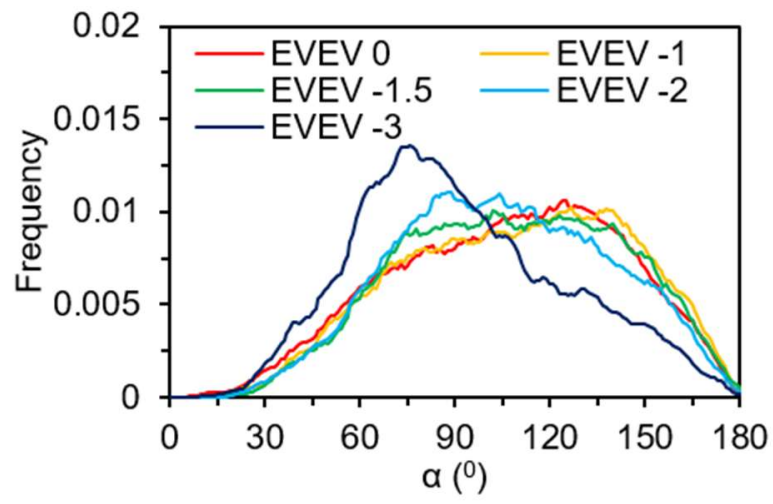

d)

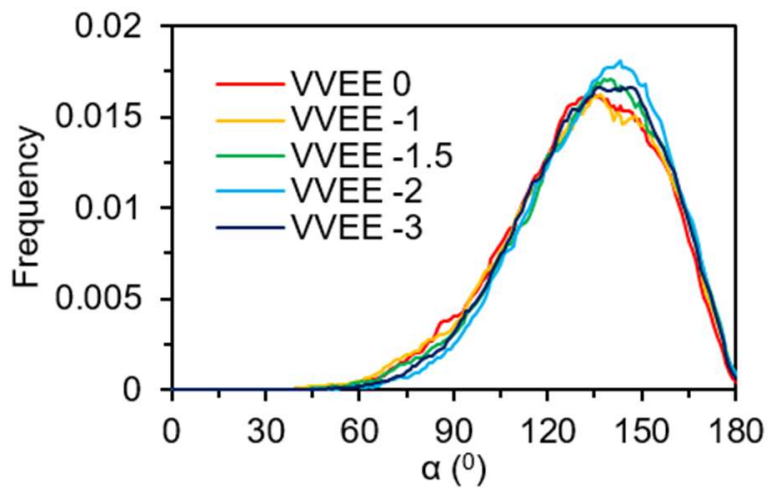

Figure S10. PA extension analysis of the fiber equilibration simulations for the four tetrapeptide isomers (a) EEVV, (b) EVEV, (c) VEVE and (d) VVEE, at different charge/PA. 


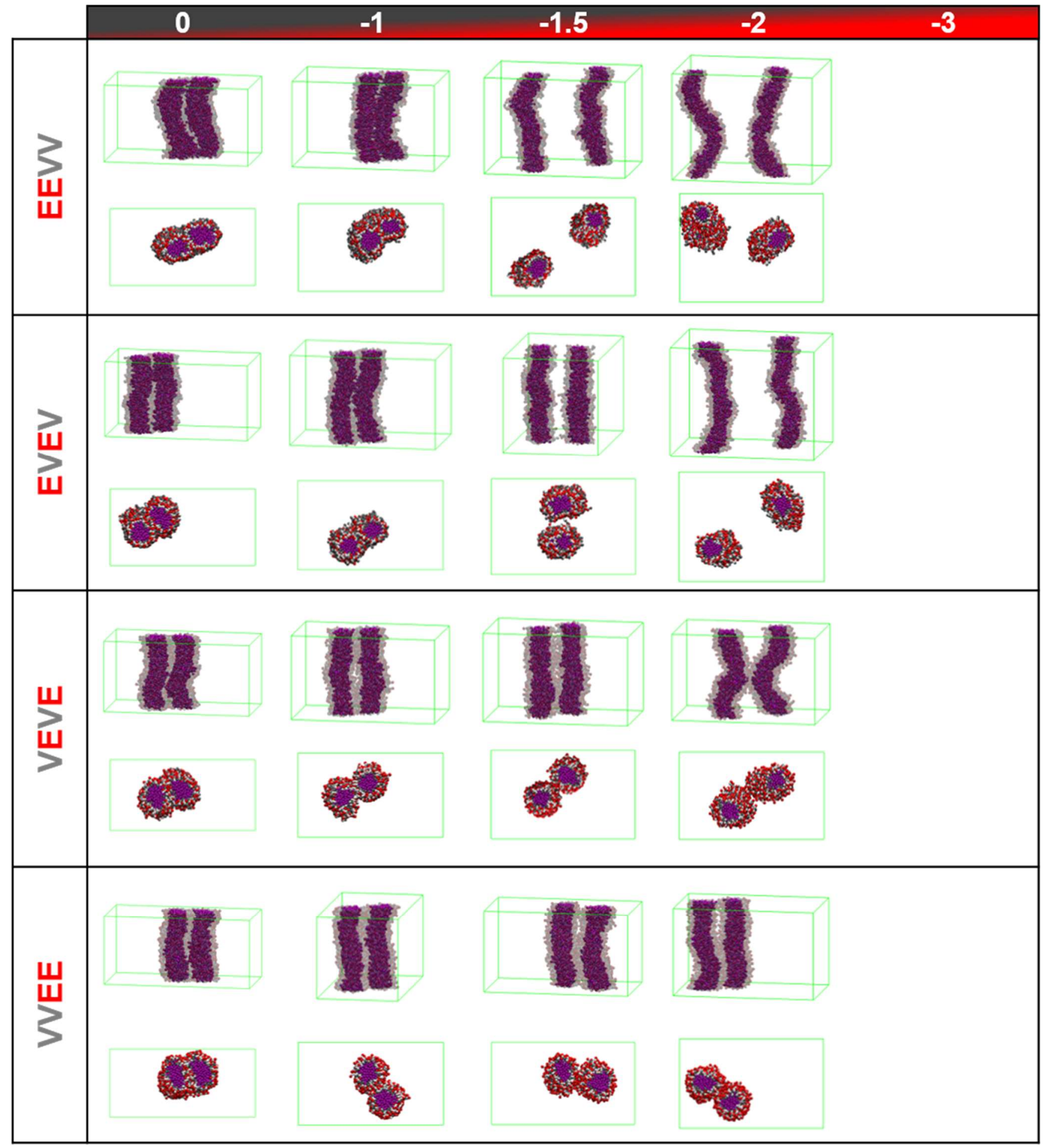

Figure S11. Final structures of the simulations of two fibers for the four isomers EEVV, EVEV, VEVE and VVEE at different charge/PA. Structures are shown with the aliphatic tail in purple, peptide backbone in white, hydrophobic side chains in grey, and hydrophilic side chains in red. Simulation box is shown in green. Water and ions are omitted for clarity. For each PA top row shows peptide semi-transparent and bottom row shows top view. 
a)

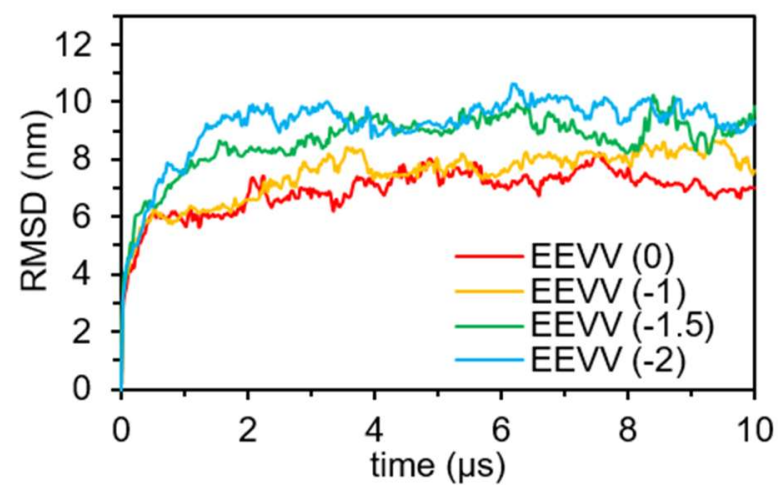

c)

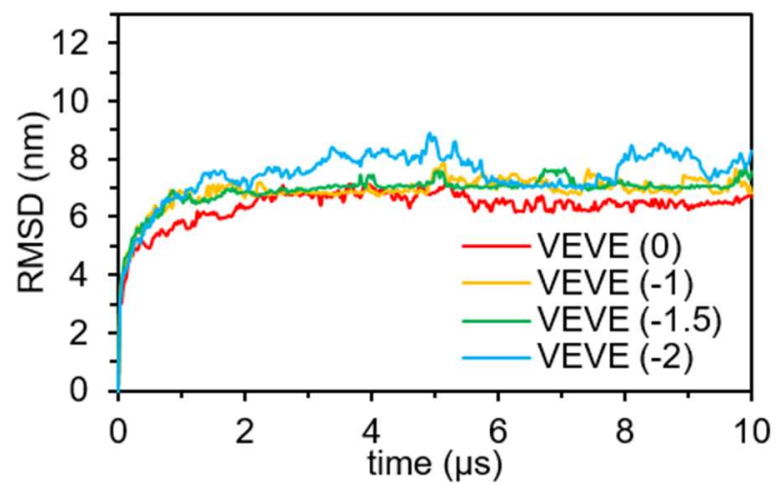

b)

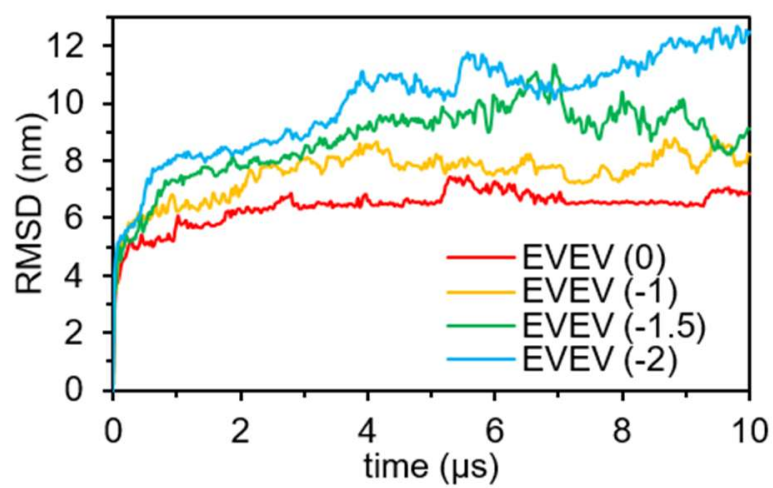

d)

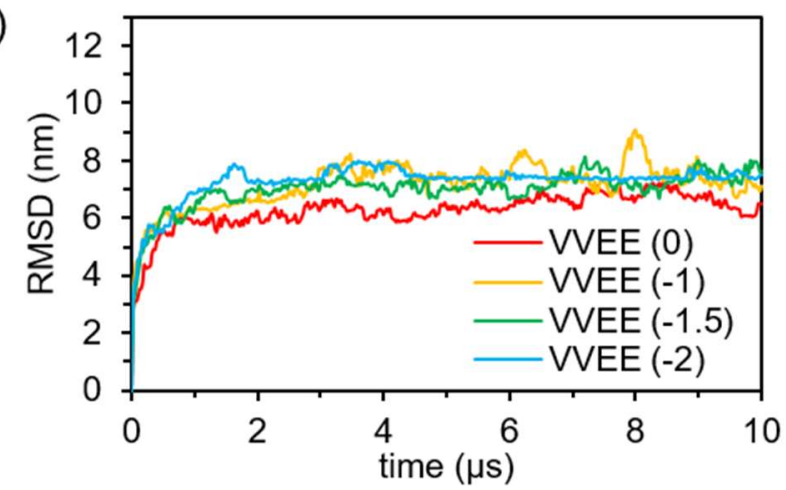

Figure S12. RMSD analysis of the simulations of two fibers for the tetrapeptide isomers (a) EEVV, (b) EVEV, (c) VEVE and (d) VVEE, at different charge/PA.

a)

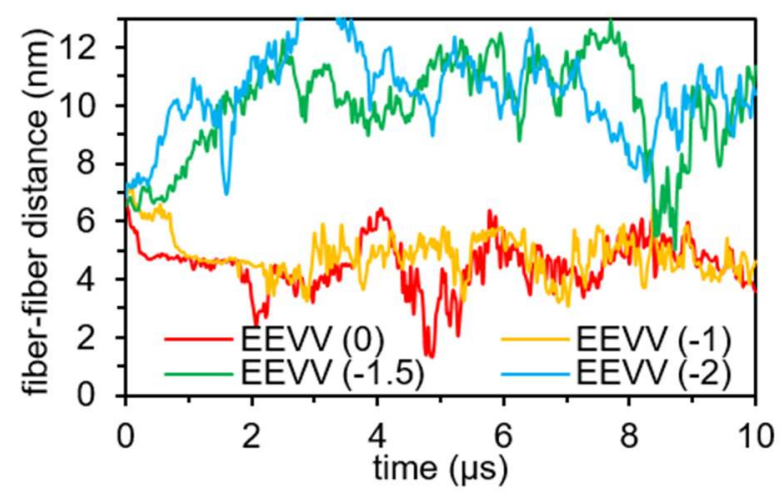

c)

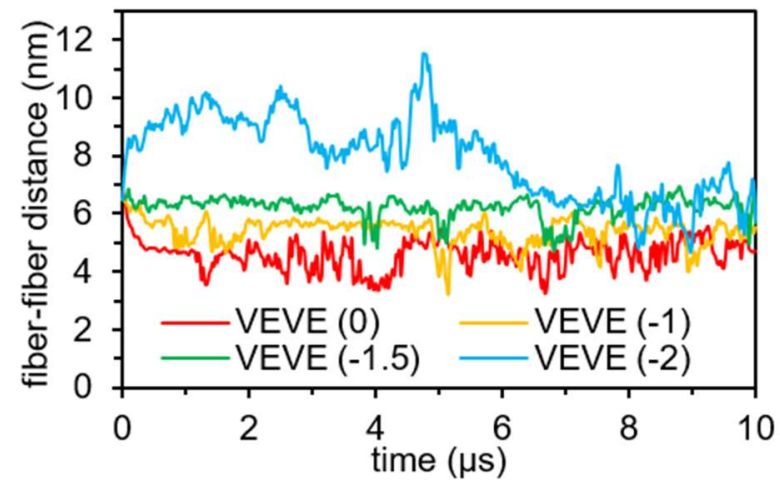

b)

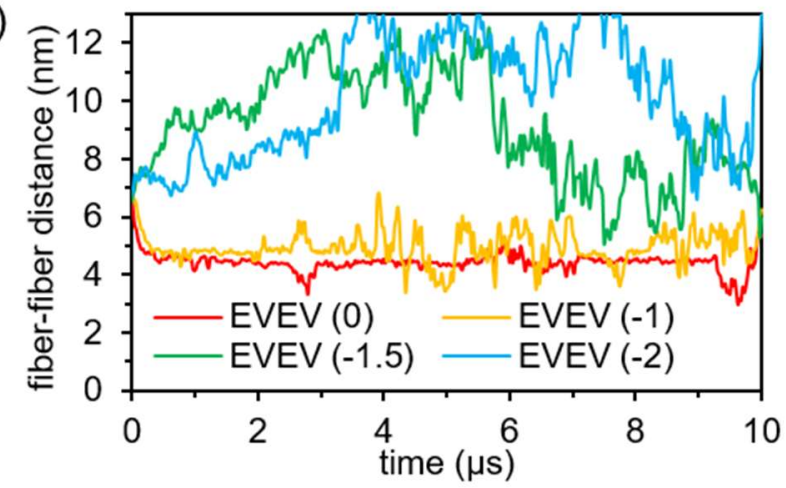

d)

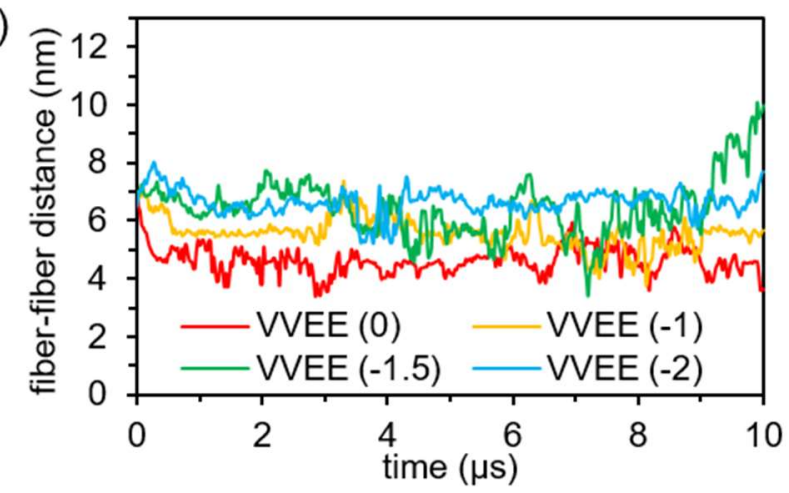

Figure S13. Interfiber distance analysis of the simulations of two fibers for the tetrapeptide isomers (a) EEVV, (b) EVEV, (c) VEVE and (d) VVEE, at different charge/PA. 
Experimental Results

PA Molecules LC-MS

a)

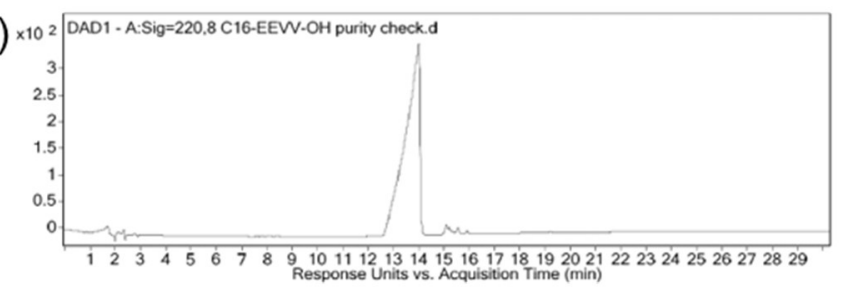

C) $\times 10^{2}+$ +ESI Scon (12.9-14.5 min, 213 scans) Frog=140.0V C16-EEW -OH purity chock.d Doconvolutod

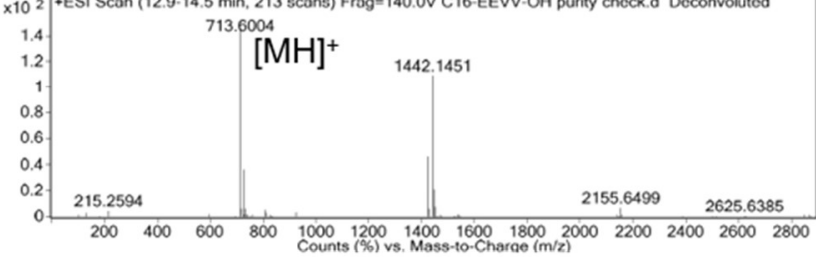

e) $\times$

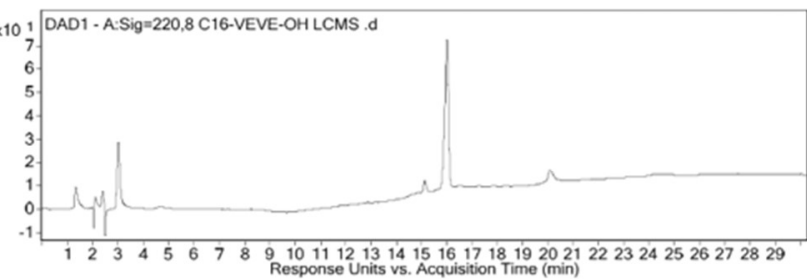

g)

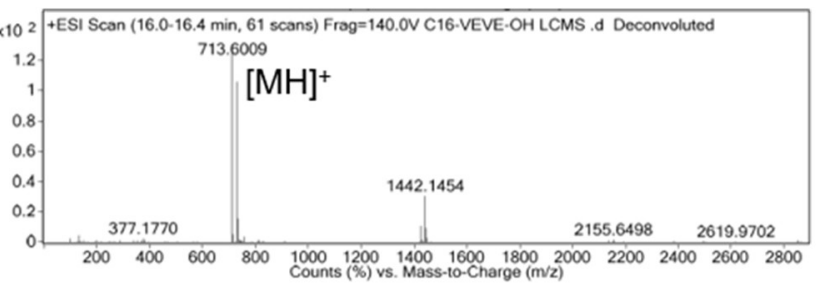

b) $\times$ (1

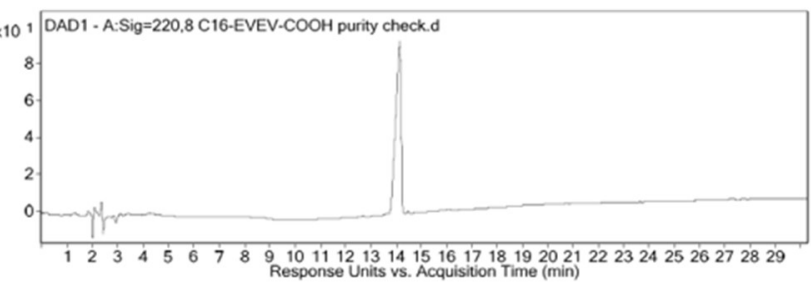

d) $\times 10$

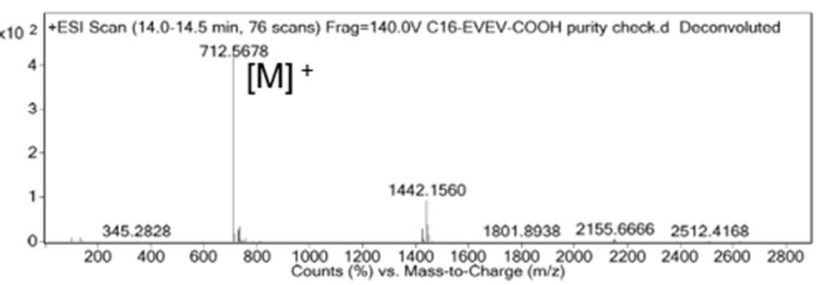

f)

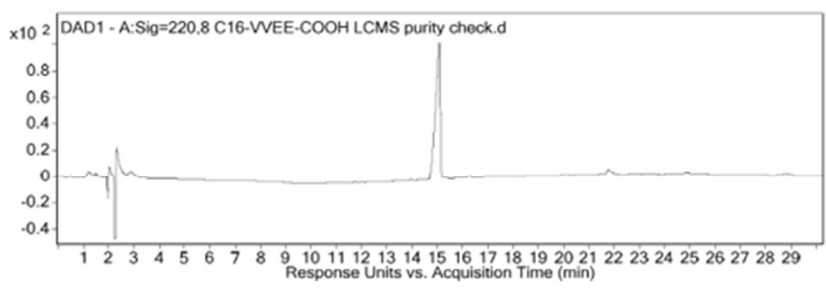

h) $\times 10^{2}+$ +ESI Scan (15.0-15.5 min, 66 scans) Frag=140.0V C16-WEE-COOH LCMS purity check.d Decon..

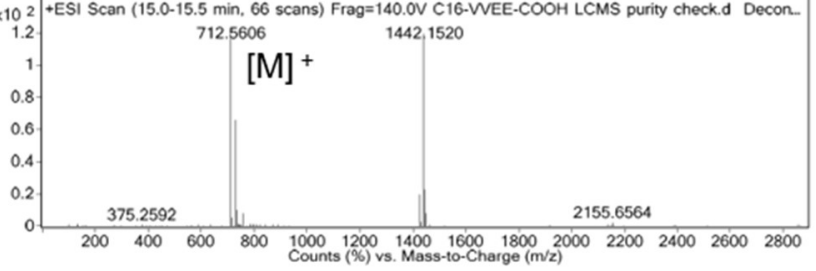

Figure S14. LC-MS results of the tetrapeptide isomers (calculated mass, $M=712.46)$ showing ( $a, b$, e, f) the chromatograms and (c, d, g, h) the m/z plots for (a, c) EEVV, (b, d) EVEV, (e, g) VEVE, and (f, h) VVEE. 


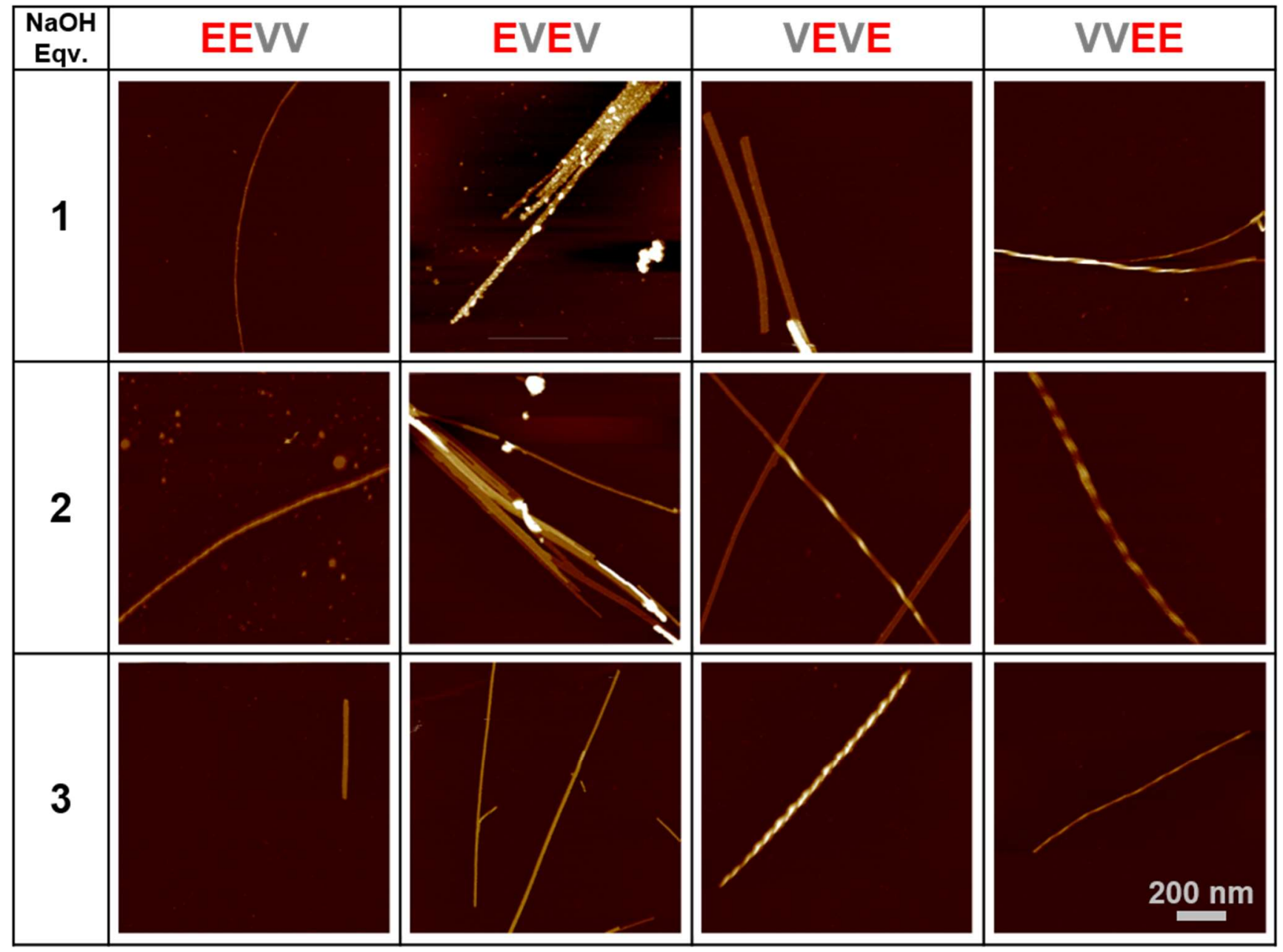

$20 \mathrm{~nm}$

Figure S15. AFM images of the four tetrapeptide isomers with addition of 1, 2 or 3 equiv. $\mathrm{NaOH}$.

a)

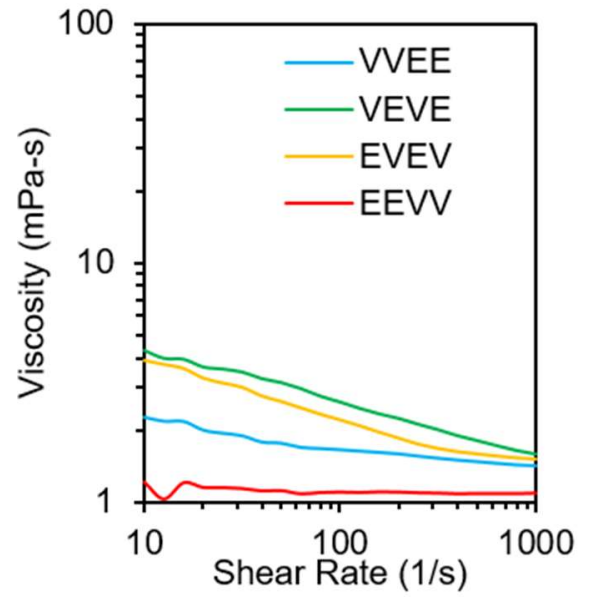

b)

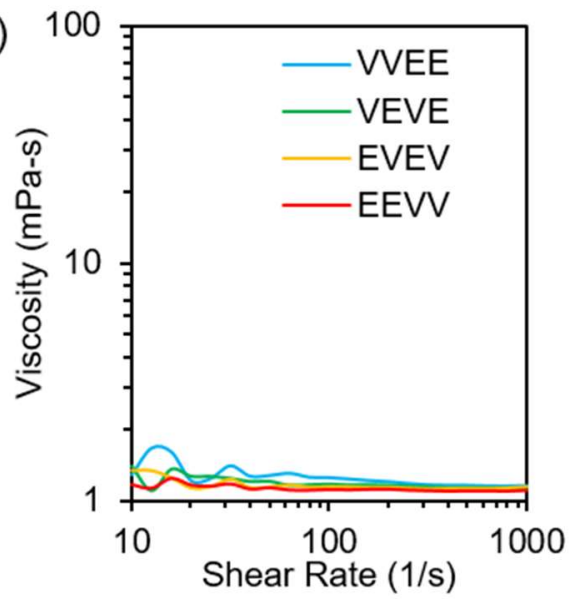

Figure S16. Viscosity curves of the four tetrapeptide isomers at (a) 2 equiv. and (b) 3 equiv. $\mathrm{NaOH}$. 


\section{References}

1. Hanwell, M. D.; Curtis, D. E.; Lonie, D. C.; Vandermeersch, T.; Zurek, E.; Hutchison, G. R. Avogadro: an advanced semantic chemical editor, visualization, and analysis platform. J. Cheminform., 2012, 4, 17.

2. Marrink, S. J.; Risselada, H. J.; Yefimov, S.; Tieleman, D. P.; de Vries, A. H. The MARTINI Force Field: Coarse Grained Model for Biomolecular Simulations. J. Phys. Chem. B, 2007, 111, 7812-7824.

3. Monticelli, L.; Kandasamy, S. K.; Periole, X.; Larson, R. G.; Tieleman, D. P.; Marrink, S.-J. The MARTINI Coarse-Grained Force Field: Extension to Proteins. J. Chem. Theory Comput., 2008, 4, 819-834.

4. de Jong, D. H.; Singh, G.; Bennett, W. F. D.; Arnarez, C.; Wassenaar, T. A.; Schäfer, L. V.; Periole, X.; Tieleman, D. P.; Marrink, S. J. Improved Parameters for the Martini Coarse-Grained Protein Force Field. J. Chem. Theory Comput., 2013, 9, 687-697.

5. $\quad$ martinize.py, 2.0; <http://cgmartini.nl/images/tools/martinize/martinize-2.6/martinize.py>.

6. Abraham, M. J.; Murtola, T.; Schulz, R.; Páll, S.; Smith, J. C.; Hess, B.; Lindahl, E. GROMACS: High performance molecular simulations through multi-level parallelism from laptops to supercomputers. SoftwareX, 2015, 1, 19-25.

7. Berendsen, H. J. C.; van der Spoel, D.; van Drunen, R. GROMACS: A message-passing parallel molecular dynamics implementation. Comput. Phys. Commun., 1995, 91, 43-56.

8. $\quad$ Frederix, P. W. J. M.; Scott, G. G.; Abul-Haija, Y. M.; Kalafatovic, D.; Pappas, C. G.; Javid, N.; Hunt, N. T.; Ulijn, R. V.; Tuttle, T. Exploring the Sequence Space for (Tri-)peptide Self-assembly to Design and Discover New Hydrogels. Nat. Chem., 2015, 7, 30-37.

9. Guo, C.; Arnon, Z. A.; Qi, R.; Zhang, Q.; Adler-Abramovich, L.; Gazit, E.; Wei, G. Expanding the Nanoarchitectural Diversity Through Aromatic Di-and Tri-Peptide Coassembly: Nanostructures and Molecular Mechanisms. ACS Nano, 2016, 10, 8316-8324.

10. Lee, O.-S.; Cho, V.; Schatz, G. C. Modeling the Self-Assembly of Peptide Amphiphiles into Fibers Using Coarse-Grained Molecular Dynamics. Nano Lett., 2012, 12, 4907-4913.

11. Mayans, E.; Ballano, G.; Casanovas, J.; Díaz, A.; Pérez-Madrigal, M. M.; Estrany, F.; Puiggalí, J.; Cativiela, C.; Alemán, C. Self-Assembly of Tetraphenylalanine Peptides. Chem.-Eur. J., 2015, 21, 16895-16905.

12. insane.py, <http://cgmartini.nl/images/tools/insane/insane.py>.

13. Humphrey, W.; Dalke, A.; Schulten, K. VMD: Visual molecular dynamics. J. Mol. Graphics, 1996, 14, 33-38.

14. De Jong, D. H.; Baoukina, S.; Ingólfsson, H. I.; Marrink, S. J. Martini straight: boosting performance using a shorter cutoff and GPUs. Comput. Phys. Commun., 2016, 199, 1-7.

15. Berendsen, H. J. C.; Postma, J. P. M.; van Gunsteren, W. F.; DiNola, A.; Haak, J. R. Molecular dynamics with coupling to an external bath. J. Chem. Phys., 1984, 81, 3684-3690.

16. Bussi, G.; Donadio, D.; Parrinello, M. Canonical sampling through velocity rescaling. J. Chem. Phys., 2007, 126, 014101. 